\title{
Fixed points for a pair of weakly compatible mappings satisfying a new type of $\phi$ - implicit relation in $S$ - metric spaces
}

\author{
Valeriu Popa \\ "Vasile Alecsandri" University of Bacău, \\ 157 Calea Mărăşeşti, Bacău, 600115, \\ Romania \\ email: vpopa@ub.ro
}

\author{
Alina-Mihaela Patriciu \\ "Dunărea de Jos" University of Galaţi, \\ Faculty of Sciences and Environment, \\ Department of Mathematics and \\ Computer Sciences, \\ 111 Domnească Street, \\ Galaţi, 800201, Romania \\ email: Alina.Patriciu@ugal.ro
}

\begin{abstract}
The purpose of this paper is to introduce a new type of $\phi-$ implicit relation in $S$ - metric spaces and to prove a general fixed point for a pair of weakly compatible mappings, which generalize Theorems 1 , 2, 4 [23], Theorems 1-7 [13], Corollary 2.19 [13], Theorems 2.2, 2.4 [19], Theorems 3.2, 3.3, 3.4 [20] and other known results.
\end{abstract}

\section{Introduction}

Let $X$ be a nonempty set and $f, g: X \rightarrow X$ two self mappings. A point $x \in X$ is said to be a coincidence point of $f$ and $g$ if $f x=g x=w$. The set of all coincidence points of $f$ and $g$ is denoted $\mathcal{C}(f, g)$ and $w$ is said to be a point of coincidence of $f$ and $g$.

In [8], Jungck defined $f$ and $g$ to be weakly compatible if $f g x=g f x$, for all $x \in \mathcal{C}(f, g)$.

2010 Mathematics Subject Classification: 54H25, 47H10

Key words and phrases: $S$ - metric space, fixed point, weakly compatible mappings, $\phi$ implicit relation 
The notion of weakly compatible mappings is used to proof the existence of common fixed point for pairs of mappings.

A new class of generalized metric space, named D - metric space, is introduced in $[5,6]$. In $[11,12]$, Mustafa and Sims proved that most of the claims concerning the fundamental topological structures on D - metric spaces are incorrect and introduced a new generalized metric spaces, named G - metric space. There exists a vast literature in the study of fixed points in $G$ - metric spaces.

In [10], Mustafa initiated the study of fixed points for weakly compatible mappings in $\mathrm{G}$ - metric spaces.

Recently in [22], the authors introduced a new class of generalized metric space, named S - metric space. Quite recently in [7], the authors proved that the notions of $\mathrm{G}$ - metric spaces and $S$ - metric space are independent.

Other results in the study of fixed points in $S$ - metric space are obtained in $[13,19,20,21]$ and in other papers. Some results of fixed points for weakly compatible mappings in $S$ - metric spaces are obtained in $[23,2]$.

In $[14,15]$, several classical fixed point theorems and common fixed point theorems have been unified considering a general condition by implicit function.

The study of fixed point for mappings satisfying an implicit relation in G metric spaces is initiated in $[16,17]$ and in other papers.

The notion of $\phi$ - maps is introduced in [9]. In [3], Altun and Turkoglu introduced a new class of implicit relation satisfying a $\phi-$ map.

A general fixed point theorem for mappings satisfying $\phi$ - implicit relations in $\mathrm{G}$ - metric spaces is obtained in [18].

The purpose of this paper is to introduce a new type of $\phi$ - implicit relation in $S$ - metric spaces and to prove a general fixed point theorem for a pair of weakly compatible mappings in $S$ - metric spaces, generalizing Theorems 1,2 , 4 [23], Theorems 1-7 [13], Corollary 2.19 [13], Theorems 2.2, 2.4 [19], Theorems $3.2,3.3,3.4[20]$ and other known results.

\section{Preliminaries}

Definition 1 ([21, 22]) A S - metric on a nonempty set $\mathrm{X}$ is a function $S: X^{3} \rightarrow \mathbb{R}_{+}$such that for all $x, y, z, a \in X:$

$\left(S_{1}\right): S(x, y, z)=0$ if and only if $x=y=z$;

$\left(S_{2}\right): S(x, y, z) \leq S(x, x, a)+S(y, y, a)+S(z, z, a)$.

The pair $(\mathrm{X}, \mathrm{S})$ is called a $\mathrm{S}$ - metric space. 
Example 1 Let $X=\mathbb{R}$ and $S(x, y, z)=|x-z|+|y-z|$. Then, $S(x, y, z)$ is a $\mathrm{S}$ - metric on $\mathbb{R}$ and is named the usual $\mathrm{S}$ - metric on $\mathrm{X}$.

Lemma $1([4,5])$ If $S$ is a $S$ - metric on a nonempty set $\mathrm{X}$, then

$$
S(x, x, y)=S(y, y, x) \text { for all } x, y \in X .
$$

Definition $2([22])$ Let $(X, S)$ be a $S$ - metric space. For $r>0$ and $x \in X$ we define the open ball with center $x$ and radius $r$, denoted $\mathrm{B}_{\mathrm{S}}(\mathrm{x}, \mathrm{r})$, respectively closed ball, denoted $\overline{\mathrm{B}}_{\mathrm{S}}(\mathrm{x}, \mathrm{r})$, the sets:

$$
B_{S}(x, r)=\{y \in X: S(x, x, y)<r\},
$$

respectively,

$$
\bar{B}_{S}(x, r)=\{y \in X: S(x, x, y) \leq r\}
$$

The topology induced by $S$ - metric on $X$ is the topology determined by the base of all open balls in $\mathrm{X}$.

Definition $3([22])$ a) A sequence $\left\{x_{n}\right\}$ in a $\mathrm{S}$ - metric space $(\mathrm{X}, \mathrm{S})$ is convergent to $x$, denoted $x_{n} \rightarrow x$ or $\lim _{n \rightarrow \infty} x_{n}=x$, if $S\left(x_{n}, x_{n}, x\right) \rightarrow 0$ as $\mathrm{n} \rightarrow \infty$, that is, for $\varepsilon>0$, there exists $\mathrm{n}_{0} \in \mathbb{N}$ such that for all $\mathrm{n} \geq \mathrm{n}_{0}$ we have $S\left(x_{n}, x_{n}, x\right)<\varepsilon$.

b) A sequence $\left\{x_{n}\right\}$ in $(X, S)$ is a Cauchy sequence if $S\left(x_{n}, x_{n}, x_{m}\right) \rightarrow 0$ as $\mathrm{n}, \mathrm{m} \rightarrow \infty$, that is, for $\varepsilon>0$, there exists $\mathrm{n}_{0} \in \mathbb{N}$ such that for all $\mathrm{m}, \mathrm{n} \geq \mathrm{n}_{0}$ we have $\mathrm{S}\left(\mathrm{x}_{\mathrm{n}}, \mathrm{x}_{\mathrm{n}}, \mathrm{x}_{\mathrm{m}}\right)<\varepsilon$.

c) A S - metric space $(\mathrm{X}, \mathrm{S})$ is complete if every Cauchy sequence is convergent.

Example 2 (X, S) by Example 1 is complete.

Lemma $2([22])$ Let $(\mathrm{X}, \mathrm{S})$ be a $\mathrm{S}$ - metric space. If $\mathrm{x}_{\mathrm{n}} \rightarrow \mathrm{x}$ and $\mathrm{y}_{\mathrm{n}} \rightarrow \mathrm{y}$, then $S\left(x_{n}, x_{n}, y_{n}\right) \rightarrow S(x, x, y)$.

Lemma $3([22])$ Let $(\mathrm{X}, \mathrm{S})$ be a $\mathrm{S}$ - metric space and $\mathrm{x}_{\mathrm{n}} \rightarrow \mathrm{x}$. Then $\lim _{\mathfrak{n} \rightarrow \infty} \mathrm{x}_{\mathrm{n}}$ is unique.

Lemma $4([4])$ Let $(\mathrm{X}, \mathrm{S})$ be a $\mathrm{S}$ - metric space and $\left\{\mathrm{x}_{\mathrm{n}}\right\}$ be a sequence in $\mathrm{X}$ such that

$$
\lim _{n \rightarrow \infty} S\left(x_{n}, x_{n}, x_{n+1}\right)=0 .
$$


If $\left\{x_{n}\right\}$ is not a Cauchy sequence, then there exists an $\varepsilon>0$ and two sequences $\left\{m_{k}\right\}$ and $\left\{n_{k}\right\}$ of positive integers with $n_{k}>m_{k}>k$ such that

$$
S\left(x_{m_{k}}, x_{m_{k}}, x_{n_{k}}\right) \geq \varepsilon, S\left(x_{m_{k-1}}, x_{m_{k-1}}, x_{n_{k}}\right)<\varepsilon
$$

and

(i) $\lim _{n \rightarrow \infty} S\left(x_{m_{k}}, x_{m_{k}}, x_{n_{k}}\right)=\varepsilon$,

(ii) $\lim _{n \rightarrow \infty} S\left(x_{m_{k}}, x_{m_{k}}, x_{n_{k-1}}\right)=\varepsilon$,

(iii) $\lim _{n \rightarrow \infty} S\left(x_{m_{k-1}}, x_{m_{k-1}}, x_{n_{k}}\right)=\varepsilon$,

(iv) $\lim _{\mathfrak{n} \rightarrow \infty} \mathrm{S}\left(x_{m_{k-1}}, x_{m_{k-1}}, x_{n_{k-1}}\right)=\varepsilon$.

Definition $4([9])$ Let $\Phi$ be the set of all functions such that $\phi:[0, \infty) \rightarrow$ $[0, \infty)$ is a nondecreasing function satisfying $\lim _{\mathfrak{n} \rightarrow \infty} \phi^{\mathrm{n}}(\mathrm{t})=0$ for all $\mathrm{t} \in$ $[0, \infty)$. If $\phi \in \Phi$, then $\phi$ is called $\phi$ - mapping. Furthermore, if $\phi \in \Phi$, then:

(i) $\phi(\mathrm{t})<\mathrm{t}$ for all $\mathrm{t} \in(0, \infty)$,

(ii) $\phi(0)=0$.

The following theorems are recently published in [23].

Theorem 1 (Theorem 1 [23]) Let (X,S) be a S - metric space. Suppose that the mappings $\mathrm{f}, \mathrm{g}: \mathrm{X} \rightarrow \mathrm{X}$ satisfy

$$
S(f x, f y, g z) \leq \phi(\max \{S(g x, g x, f x), S(g y, g y, f y), S(g z, g z, f z)\})
$$

for all $x, y, z \in X$.

If $\mathrm{f}(\mathrm{X}) \subset \mathrm{g}(\mathrm{X})$ and one of $\mathrm{f}(\mathrm{X})$ or $\mathrm{g}(\mathrm{X})$ is a complete subspace of $\mathrm{X}$, then $f$ and $g$ have a unique point of coincidence.

Moreover, if $\mathrm{f}$ and $\mathrm{g}$ are weakly compatible, then $\mathrm{f}$ and $\mathrm{g}$ have a unique common fixed point.

Theorem 2 (Theorem 2 [23]) Let $(\mathrm{X}, \mathrm{S})$ be a $\mathrm{S}$ - metric space. Suppose that the mappings $\mathrm{f}, \mathrm{g}: \mathrm{X} \rightarrow \mathrm{X}$ satisfy

$$
S(f x, f y, f z) \leq \max \{\phi(S(g x, g x, f x)), \phi(S(g y, g y, f y)), \phi(S(g z, g z, f z))\}
$$

for all $x, y, z \in X$.

If $\mathrm{f}(\mathrm{X}) \subset \mathrm{g}(\mathrm{X})$ and one of $\mathrm{f}(\mathrm{X})$ or $\mathrm{g}(\mathrm{X})$ is a complete subspace of $\mathrm{X}$, then $\mathrm{f}$ and $\mathrm{g}$ have a unique point of coincidence. Moreover, if $\mathrm{f}$ and $\mathrm{g}$ are weakly compatible, then $\mathrm{f}$ and $\mathrm{g}$ have a unique common fixed point. 
Theorem 3 (Theorem 4 [23]) Let $(\mathrm{X}, \mathrm{S})$ be a $\mathrm{S}$ - metric space. Suppose that the mappings $\mathrm{f}, \mathrm{g}: \mathrm{X} \rightarrow \mathrm{X}$ satisfy

$S(f x, f y, f z) \leq k_{1} \phi(S(g x, g x, f x))+k_{2} \phi(S(g y, g y, f y))+k_{3} \phi(S(g z, g z, f z))$ for all $x, y, z \in X, k_{1}+k_{2}+k_{3}<1$.

If $\mathrm{f}(\mathrm{X}) \subset \mathrm{g}(\mathrm{X})$ and one of $\mathrm{f}(\mathrm{X})$ or $\mathrm{g}(\mathrm{X})$ is a complete subspace of $\mathrm{X}$, then $\mathrm{f}$ and $\mathrm{g}$ have a unique point of coincidence. Moreover, if $\mathrm{f}$ and $\mathrm{g}$ are weakly compatible, then $\mathrm{f}$ and $\mathrm{g}$ have a unique common fixed point.

Remark 1 1) Since $\phi(t)$ is nondecreasing, then

$\phi\left(\max \left\{t_{2}, t_{3}, t_{4}, t_{5}, t_{6}\right\}\right)=\max \left\{\phi\left(t_{2}\right), \phi\left(t_{3}\right), \phi\left(t_{4}\right), \phi\left(t_{5}\right), \phi\left(t_{6}\right)\right\}$.

Hence, Theorem 2 is Theorem 1.

2) By (3) we obtain

$$
\begin{aligned}
S(f x, f y, f z) \leq & \left(k_{1}+k_{2}+k_{3}\right) \max \{\phi(S(g x, g x, f x)), \\
& \phi(S(g y, g y, f y)), \phi(S(g z, g z, f z))\} \\
= & \left(k_{1}+k_{2}+k_{3}\right) \phi(\max \{S(g x, g x, f x), S(g y, g y, f y), \\
& S(g z, g z, f z)\}) \\
\leq & \phi(\max \{\max \{S(g x, g x, f x), S(g y, g y, f y), S(g z, g z, f z)\}\}) .
\end{aligned}
$$

Hence,

$$
S(f x, f y, f z) \leq \phi(\max \{S(g x, g x, f x), S(g y, g y, f y), S(g z, g z, f z)\}),
$$

which is the inequality (1). Hence, Theorem 3 is a particular case of Theorem 1.

3) In the proof of Theorem 1 is used $\mathrm{x}=\mathrm{y}$. Hence in Theorem 1 we have a new form of inequality (1):

$$
S(f x, f x, f y) \leq \phi(\max \{S(g x, g x, f x), g(f y, g y, f y)\}) .
$$

\section{$3 \quad \phi$ - implicit relations}

Let $\mathcal{F}_{\phi}$ be the set of all lower semi - continuous functions $\mathrm{F}: \mathbb{R}_{+}^{6} \rightarrow \mathbb{R}$ such that:

$\left(F_{1}\right): \quad F$ is nonincreasing in variable $t_{6}$, 
$\left(F_{2}\right): \quad$ There exists $\phi \in \mathcal{F}_{\phi}$ such that for all $u, v \geq 0, F(u, v, v, u, 0,2 u+v) \leq$ 0 implies $u \leq \phi(v)$

$\left(F_{3}\right): \quad F(t, t, 0,0, t, t)>0, \forall t>0$.

In all the following examples, $\left(F_{1}\right)$ is obviously.

Example $3 F\left(t_{1}, \ldots, t_{6}\right)=t_{1}-k \max \left\{t_{2}, t_{3}, \ldots, t_{6}\right\}$, where $k \in\left[0, \frac{1}{3}\right)$.

$\left(F_{2}\right):$ Let $u, v \geq 0$ and $F(u, v, v, u, 0,2 u+v)=u-k(u+2 v) \leq 0$. If $u>v$, then $u(1-3 k) \leq 0$, a contradiction. Hence, $u \leq v$, which implies $u \leq 3 k v$ and $F$ satisfies $\left(F_{2}\right)$ for $\phi(t)=3 k t$.

$\left(F_{3}\right): \quad F(t, t, 0,0, t, t)=t(1-k)>0, \forall t>0$.

Example $4 \mathrm{~F}\left(\mathrm{t}_{1}, \ldots, \mathrm{t}_{6}\right)=\mathrm{t}_{1}-\mathrm{k} \max \left\{\mathrm{t}_{2}, \mathrm{t}_{3}, \mathrm{t}_{4}, \frac{\mathrm{t}_{5}+\mathrm{t}_{6}}{3}\right\}$, where $\mathrm{k} \in[0,1)$.

$\left(F_{2}\right): \quad$ Let $u, v \geq 0$ and $F(u, v, v, u, 0,2 u+v)=u-k \max \left\{u, v, \frac{2 u+v}{3}\right\} \leq$ 0 . If $u>v$, then $u(1-k) \leq 0$, a contradiction. Hence, $u \leq v$, which implies $u \leq k v$ and $F$ satisfies $\left(F_{2}\right)$ for $\phi(t)=k t$.

$\left(F_{3}\right): \quad F(t, t, 0,0, t, t)=t(1-k)>0, \forall t>0$.

Example $5 \mathrm{~F}\left(\mathrm{t}_{1}, \ldots, \mathrm{t}_{6}\right)=\mathrm{t}_{1}-a t_{2}-b t_{3}-\mathrm{ct}_{4}-d t_{5}-e t_{6}$, where $\mathrm{a}, \mathrm{b}, \mathrm{c}, \mathrm{d}, \mathrm{e} \geq 0$ and $\mathrm{a}+\mathrm{b}+\mathrm{c}+3 \mathrm{e}<1$ and $\mathrm{a}+\mathrm{d}+\mathrm{e}<1$.

$\left(F_{2}\right)$ : Let $u, v \geq 0$ and $F(u, v, v, u, 0,2 u+v)=u-a v-b v-c u-$ $e(2 u+v) \leq 0$. If $u>v$, then $u[1-(a+b+c+3 e)] \leq 0$, a contradiction. Hence, $u \leq v$, which implies $u \leq(a+b+c+3 e) v$ and $F$ satisfies $\left(F_{2}\right)$ for $\phi(t)=(a+b+c+3 e) t$.

$\left(F_{3}\right): \quad F(t, t, 0,0, t, t)=t[1-(a+d+e)]>0, \forall t>0$.

Example $6 \mathrm{~F}\left(\mathrm{t}_{1}, \ldots, \mathrm{t}_{6}\right)=\mathrm{t}_{1}^{2}-\mathrm{t}_{1}\left(\mathrm{at}_{2}+\mathrm{bt}_{3}+\mathrm{ct}_{4}\right)-\mathrm{dt}_{5} \mathrm{t}_{6}$, where $\mathrm{a}, \mathrm{b}, \mathrm{c}, \mathrm{d} \geq$ $0, a+b+c<1$ and $a+d<1$.

$\left(F_{2}\right)$ : Let $u, v \geq 0$ and $F(u, v, v, u, 0,2 u+v)=u^{2}-u(a v+b v+c u) \leq 0$. If $u>v$, then $u^{2}[1-(a+b+c)] \leq 0$, a contradiction. Hence, $u \leq v$, which implies $u \leq(a+b+c) v$ and $F$ satisfies $\left(F_{2}\right)$ for $\phi(t)=(a+b+c) t$.

$\left(F_{3}\right): \quad F(t, t, 0,0, t, t)=t^{2}[1-(a+d)]>0, \forall t>0$.

Example $7 \mathrm{~F}\left(\mathrm{t}_{1}, \ldots, \mathrm{t}_{6}\right)=\mathrm{t}_{1}^{2}-\mathrm{at}_{2}^{2}-\frac{\mathrm{bt}_{5} \mathrm{t}_{6}}{1+\mathrm{t}_{3}^{2}+\mathrm{t}_{4}^{2}}$, where $\mathrm{a}, \mathrm{b} \geq 0$ and $\mathrm{a}+\mathrm{b}<1$. 
$\left(F_{2}\right)$ : Let $u, v \geq 0$ and $F(u, v, v, u, 0,2 u+v)=u^{2}-a v^{2} \leq 0$, which implies $u \leq \sqrt{a} v$. Hence, $F$ satisfies $\left(F_{2}\right)$ for $\phi(t)=\sqrt{a} t$.

$\left(F_{3}\right): \quad F(t, t, 0,0, t, t)=t^{2}[1-(a+b)]>0, \forall t>0$.

In the following examples, if $\phi \in \Phi$, then $F$ satisfy properties $\left(F_{1}\right),\left(F_{2}\right),\left(F_{3}\right)$.

Example $8 F\left(t_{1}, \ldots, t_{6}\right)=t_{1}-\phi\left(\max \left\{t_{2}, t_{3}, t_{4}, \frac{t_{5}+t_{6}}{3}\right\}\right)$.

$\left(F_{2}\right)$ : Let $u, v \geq 0$ and

$$
F(u, v, v, u, 0,2 u+v)=u-\phi\left(\max \left\{u, v, \frac{2 u+v}{3}\right\}\right) \leq 0 .
$$

If $u>v$, then $u \leq \phi(u)<u$, a contradiction. Hence, $u \leq v$, which implies $u \leq \phi(v)$.

$\left(F_{3}\right): \quad F(t, t, 0,0, t, t)=t-\phi(t)>0, \forall t>0$.

Example $9 F\left(t_{1}, \ldots, t_{6}\right)=t_{1}-\phi\left(\max \left\{t_{2}, \frac{t_{3}+t_{4}}{2}, \frac{t_{5}+t_{6}}{3}\right\}\right)$.

$\left(F_{2}\right): \quad$ Let $u, v \geq 0$ and

$$
F(u, v, v, u, 0,2 u+v)=u-\phi\left(\max \left\{u, \frac{u+v}{2}, \frac{2 u+v}{3}\right\}\right) \leq 0 .
$$

If $u>v$, then $u \leq \phi(u)<u$, a contradiction. Hence, $u \leq v$, which implies $u \leq \phi(v)$.

$\left(F_{3}\right): \quad F(t, t, 0,0, t, t)=t-\phi(t)>0, \forall t>0$.

Example $10 F\left(t_{1}, \ldots, t_{6}\right)=t_{1}-\phi\left(a t_{2}+b \max \left\{t_{3}, t_{4}\right\}+c \max \left\{t_{5}, t_{6}\right\}\right)$, where $a, b, c \geq 0$ and $a+b+3 c<1$.

$\left(F_{2}\right):$ Let $u, v \geq 0$ and

$$
F(u, v, v, u, 0,2 u+v)=u-\phi(a v+b \max \{u, v\}+c(2 u+v)) \leq 0 .
$$

If $u>v$, then $u-\phi((a+b+3 c) u) \leq 0$, which implies $u \leq \phi(u)<u$, a contradiction. Hence, $u \leq v$ and $u \leq \phi(v)$.

$\left(F_{3}\right): \quad F(t, t, 0,0, t, t)=t-\phi(a t+c t) \geq t-\phi((a+b+3 c) t) \geq t-$ $\phi(t)>0, \forall t>0$.

Example $11 F\left(t_{1}, \ldots, t_{6}\right)=t_{1}-\phi\left(a \sqrt{t_{1} t_{2}}+b \sqrt{t_{3} t_{4}}+c \sqrt{t_{5} t_{6}}\right)$, where $a, b, c$ $\geq 0$ and $\mathrm{a}+\mathrm{b}+\mathrm{c}<1$. 
$\left(F_{2}\right): \quad$ Let $u, v \geq 0$ and $F(u, v, v, u, 0,2 u+v)=u-\phi(a \sqrt{u v}+b \sqrt{u v}) \leq 0$. If $u>v$, then $u \leq \phi((a+b) u)<u$, a contradiction. Hence, $u \leq v$, which implies $u \leq \phi(v)$.

$\left(F_{3}\right): \quad F(t, t, 0,0, t, t)=t-\phi((a+c) t) \geq t-\phi((a+b+c) t) \geq t-$ $\phi(t)>0, \forall t>0$.

Example $12 F\left(t_{1}, \ldots, t_{6}\right)=t_{1}-\phi\left(a t_{2}, \frac{b \sqrt{t_{5} t_{6}}}{1+t_{3}+t_{4}}\right)$, where $a, b \geq 0$ and $a+b<1$.

$\left(F_{2}\right)$ : Let $u, v \geq 0$ and $F(u, v, v, u, 0,2 u+v)=u-\phi(a v) \leq 0$. If $u>v$, then $u-\phi(a v) \leq 0$ implies $u \leq \phi(u)<u$, a contradiction. Hence, $u \leq v$, which implies $u \leq \phi(v)$.

$\left(F_{3}\right): \quad F(t, t, 0,0, t, t)=t-\phi((a+b) t) \geq t-\phi(t)>0, \forall t>0$.

In the following examples, the proofs are similar to the proof of Example 12 and thus are omitted.

Example $13 F\left(t_{1}, \ldots, t_{6}\right)=t_{1}-a t_{2}-b \max \left\{t_{3}, t_{4}, t_{5}, t_{6}\right\}$, where $a, b \geq 0$ and $a+3 b<1$.

If $F(u, v, v, u, 0,2 u+v) \leq 0$, then we have $u \leq \phi(v)$, where $\phi(t)=(a+3 b) t$.

Example $14 F\left(t_{1}, \ldots, t_{6}\right)=t_{1}-a t_{2}-b t_{3}-c t_{4}-d \max \left\{t_{5}, t_{6}\right\}$, where $a, b, c, d \geq$ 0 and $\mathrm{a}+\mathrm{b}+\mathrm{c}+3 \mathrm{~d}<1$.

If $F(u, v, v, u, 0,2 u+v) \leq 0$ then we have $u \leq \phi(v)$, where $\phi(t)=(a+b+$ $c+3 d) t$.

Example $15 F\left(t_{1}, \ldots, t_{6}\right)=t_{1}-a t_{2}-d \max \left\{t_{3}, t_{4}\right\}-b t_{5}-c_{6}$, where $a, b, c, d \geq$ $0, a+3 c+d \geq 0, a+3 c+d<1$ and $a+b+c<1$.

If $F(u, v, v, u, 0,2 u+v) \leq 0$ then $u \leq \phi(v)$, where $\phi(t)=(a+3 c+d) t$.

Example $16 F\left(t_{1}, \ldots, t_{6}\right)=t_{1}-a t_{2}-b t_{3}-e t_{4}-c t_{5}-d t_{6}-f \max \left\{t_{2}, t_{3}, \ldots, t_{6}\right\}$, where $a, b, c, d, e, f \geq 0, a+b+e+3 d+3 f<1$ and $a+c+e+f<1$.

If $F(u, v, v, u, 0,2 u+v) \leq 0$ then $u \leq \phi(v)$, where $\phi(t)=(a+b+e+3 d$ $+3 f)$ t.

Example $17 F\left(t_{1}, \ldots, t_{6}\right)=t_{1}-a\left(t_{5}+t_{6}\right)-b t_{2}-c \max \left\{t_{3}, t_{4}\right\}$, where $a, b, c \geq$ 0 and $3 a+b+c<1$. 
If $F(u, v, v, u, 0,2 u+v) \leq 0$ then $u \leq \phi(v)$, where $\phi(t)=(3 a+b+c) t$.

Example $18 F\left(t_{1}, \ldots, t_{6}\right)=t_{1}-a\left(t_{3}+t_{4}\right)-b t_{2}-c \max \left\{t_{5}, t_{6}\right\}$, where $a, b, c \geq$ 0 and $2 a+b+3 c<1$.

If $F(u, v, v, u, 0,2 u+v) \leq 0$ then $u \leq \phi(v)$, where $\phi(t)=(2 a+b+3 c) t$.

Example $19 F\left(t_{1}, \ldots, t_{6}\right)=t_{1}-a \max \left\{t_{4}+t_{5}, t_{3}+t_{6}\right\}-b t_{2}$, where $a, b, c \geq 0$ and $4 \mathrm{a}+\mathrm{b}<1$.

If $F(u, v, v, u, 0,2 u+v) \leq 0$ then $u \leq \phi(v)$, where $\phi(t)=(4 a+b) t$.

\section{Main results}

Lemma 5 ([1]) Let $\mathbf{f}$ and $\mathrm{g}$ be weakly compatible self mappings of a nonempty set $\mathrm{X}$. If $\mathrm{f}$ and $\mathrm{g}$ have a unique point of coincidence $\boldsymbol{w}=\mathrm{fx}=\mathrm{gx}$ for some $\mathrm{x} \in \mathrm{X}$, then $\mathrm{w}$ is the unique common fixed point of $\mathrm{f}$ and $\mathrm{g}$.

Theorem 4 Let $(\mathrm{X}, \mathrm{S})$ be a $\mathrm{S}$ - metric space and $\mathrm{f}, \mathrm{g}: \mathrm{X} \rightarrow \mathrm{X}$ such that

$$
F\left(\begin{array}{l}
S(f x, f x, f y), S(g x, g x, g y), S(g x, g x, f x), \\
S(g y, g y, f y), S(g y, g y, f x), S(g x, g x, f y)
\end{array}\right) \leq 0
$$

for all $\mathrm{x}, \mathrm{y} \in \mathrm{X}$ and some $\mathrm{F} \in \mathcal{F}_{\phi}$.

If $\mathrm{f}(\mathrm{X}) \subset \mathrm{g}(\mathrm{X})$ (or $\mathrm{g}(\mathrm{X}) \subset \mathrm{f}(\mathrm{X})$ ) and $\mathrm{g}(\mathrm{X})$ (or $\mathrm{f}(\mathrm{X})$ ) is a complete subspace of $(\mathrm{X}, \mathrm{S})$, then $\mathrm{f}$ and $\mathrm{g}$ have a unique point of coincidence. Moreover, if $\mathrm{f}$ and $\mathrm{g}$ are weakly compatible, then $\mathrm{f}$ and $\mathrm{g}$ have a unique common fixed point.

Proof. Let $x_{0}$ be an arbitrary point of $X$. Since $f(X) \subset g(X)$, there exists $x_{1} \in X$ such that $f x_{0}=g x_{1}$. Continuing this process we define the sequence $\left\{x_{n}\right\}$ satisfying

$$
f x_{n}=g x_{n+1} \text { for } n \in \mathbb{N} .
$$

Then, by (4) for $x=x_{n-1}$ and $y=x_{n}$ we have

$$
\begin{gathered}
F\left(\begin{array}{c}
S\left(f x_{n-1}, f x_{n-1}, f x_{n}\right), S\left(g x_{n-1}, g x_{n-1}, g x_{n}\right), S\left(g x_{n-1}, g x_{n-1}, f x_{n-1}\right), \\
S\left(g x_{n}, g x_{n}, f x_{n}\right), S\left(g x_{n}, g x_{n}, f x_{n-1}\right), S\left(g x_{n-1}, g x_{n-1}, f x_{n}\right)
\end{array}\right) \leq 0 \\
F\left(\begin{array}{c}
S\left(g x_{n}, g x_{n}, g x_{n+1}\right), S\left(g x_{n-1}, g x_{n-1}, g x_{n}\right), S\left(g x_{n-1}, g x_{n-1}, g x_{n}\right), \\
S\left(g x_{n}, g x_{n}, g x_{n+1}\right), 0, S\left(g x_{n-1}, g x_{n-1}, g x_{n+1}\right)
\end{array}\right) \leq 0
\end{gathered}
$$


By $\left(S_{2}\right)$ and Lemma 1 we have

$$
S\left(g x_{n-1}, g x_{n-1}, g x_{n+1}\right) \leq 2 S\left(g x_{n}, g x_{n}, g x_{n+1}\right)+S\left(g x_{n-1}, g x_{n-1}, g x_{n}\right) .
$$

By $(5)$ and $\left(F_{1}\right)$ we obtain

$F\left(\begin{array}{c}S\left(g x_{n}, g x_{n}, g x_{n+1}\right), S\left(g x_{n-1}, g x_{n-1}, g x_{n}\right), S\left(g x_{n-1}, g x_{n-1}, g x_{n}\right), \\ S\left(g x_{n}, g x_{n}, g x_{n+1}\right), 0,2 S\left(g x_{n}, g x_{n}, g x_{n+1}\right)+S\left(g x_{n-1}, g x_{n-1}, g x_{n}\right)\end{array}\right) \leq 0$.

By $\left(F_{2}\right)$ we obtain

$$
S\left(g x_{n}, g x_{n}, g x_{n+1}\right) \leq \phi\left(S\left(g x_{n-1}, g x_{n-1}, g x_{n}\right)\right), \text { for } n=1,2, \ldots
$$

which implies

$$
S\left(g x_{n}, g x_{n}, g x_{n+1}\right) \leq \phi^{n}\left(S\left(g x_{0}, g x_{0}, g x_{1}\right)\right) .
$$

Letting $\mathrm{n}$ tend to infinity we obtain

$$
\lim _{n \rightarrow \infty} S\left(g x_{n}, g x_{n}, g x_{n+1}\right)=0 .
$$

We prove that $\left\{g x_{n}\right\}$ is a Cauchy sequence in $g(X)$. Suppose that $\left\{g x_{n}\right\}$ is not a Cauchy sequence. Then, by Lemma 4, there exists an $\varepsilon>0$ and two sequences $m_{k}$ and $n_{k}$ with $n_{k}>m_{k}>k$ and $S\left(x_{m_{k}}, x_{m_{k}}, x_{n_{k}}\right) \geq \varepsilon$ and $\mathrm{S}\left(\mathrm{x}_{\mathrm{m}_{k}-1}, x_{\mathfrak{m}_{k}-1}, x_{\mathfrak{n}_{k}}\right)<\varepsilon$ and satisfying the inequalities (i) - (iv) by Lemma 4 .

By (4) for $x=x_{m_{k}-1}$ and $y=x_{n_{k}-1}$ we have

$$
\begin{gathered}
F\left(\begin{array}{c}
S\left(f x_{m_{k}-1}, f x_{m_{k}-1}, f x_{n_{k}-1}\right), S\left(g x_{m_{k}-1}, g x_{m_{k}-1}, g x_{n_{k}-1}\right), \\
S\left(g x_{m_{k}-1}, g x_{m_{k}-1}, f x_{m_{k}-1}\right), S\left(g x_{n_{k}-1}, g x_{n_{k}-1}, f x_{n_{k}-1}\right), \\
S\left(g x_{n_{k}-1}, g x_{n_{k}-1}, f x_{m_{k}-1}\right), S\left(g x_{m_{k}-1}, g x_{m_{k}-1}, f x_{n_{k}-1}\right)
\end{array}\right) \leq 0 \\
F\left(\begin{array}{c}
S\left(g x_{m_{k}}, g x_{m_{k}}, g x_{n_{k}}\right), S\left(g x_{m_{k}-1}, g x_{m_{k}-1}, g x_{n_{k}-1}\right), \\
S\left(g x_{m_{k}-1}, g x_{m_{k}-1}, g x_{m_{k}}\right), S\left(g x_{n_{k}-1}, g x_{n_{k}-1}, g x_{n_{k}}\right), \\
S\left(g x_{n_{k}-1}, g x_{n_{k}-1}, g x_{m_{k}}\right), S\left(g x_{m_{k}-1}, g x_{m_{k}-1}, g x_{n_{k}}\right)
\end{array}\right) \leq 0 .
\end{gathered}
$$

By Lemma 1,

$$
S\left(g x_{m_{k}-1}, g x_{m_{k}-1}, g x_{n_{k}}\right)=S\left(g x_{n_{k}}, g x_{n_{k}}, g x_{m_{k}-1}\right)
$$

and

$$
S\left(g x_{n_{k}-1}, g x_{n_{k}-1}, g x_{m_{k}}\right)=S\left(g x_{m_{k}}, g x_{m_{k}}, g x_{n_{k}-1}\right) .
$$


Letting $\mathrm{n}$ tend to infinity in (6) we obtain

$$
\mathrm{F}(\varepsilon, \varepsilon, 0,0, \varepsilon, \varepsilon) \leq 0,
$$

a contradiction of $\left(\mathrm{F}_{3}\right)$.

Hence, $\left\{g x_{n}\right\}$ is a Cauchy sequence in $g(X)$. Since $g(X)$ is complete, then $\left\{g x_{n}\right\}$ is convergent to a point $t \in g(X)$. Hence, there exists $p \in X$ such that $g p=t$ and $\lim _{n \rightarrow \infty} g x_{n}=g p$. We prove that $f p=g p$.

By (4) for $x=x_{n}$ and $y=p$ we have

$$
F\left(\begin{array}{c}
S\left(g x_{n}, g x_{n}, f p\right), S\left(g x_{n}, g x_{n}, g p\right), S\left(g x_{n}, g x_{n}, f x_{n}\right), \\
S(g p, g p, f p), S\left(g p, g p, f x_{n}\right), S\left(g x_{n}, g x_{n}, f p\right)
\end{array}\right) \leq 0 .
$$

Letting $\mathrm{n}$ tend to infinity we obtain

$$
F(S(g p, g p, f p), 0,0, S(g p, g p, f p), 0, S(g p, g p, f p)) \leq 0 .
$$

By $\left(F_{1}\right)$ we have

$$
F(S(g p, g p, f p), 0,0, S(g p, g p, f p), 0,2 S(g p, g p, f p)) \leq 0,
$$

which implies $S(g p, g p, f p)=0$. Hence $g p=f p=t$.

We prove that $t$ is the unique point of coincidence of $f$ and $g$. Suppose that there exists $z=f w=g w$. By (4) we obtain

$$
\begin{aligned}
& F\left(\begin{array}{c}
S(f p, f p, f w), S(g p, g p, g w), S(g p, g p, f p), \\
S(g w, g w, f w), S(g w, g w, f p), S(g p, g p, f w)
\end{array}\right) \leq 0, \\
& \mathrm{~F}(\mathrm{~S}(\mathrm{t}, \mathrm{t}, z), S(\mathrm{t}, \mathrm{t}, z), 0,0, S(z, z, t), S(t, t, z)) \leq 0 \text {. }
\end{aligned}
$$

By Lemma 1 we have

$$
F(S(t, t, z), S(t, t, z), 0,0, S(t, t, z), S(t, t, z)) \leq 0,
$$

a contradiction of $\left(F_{3}\right)$ if $S(t, t, z)>0$. Hence, $z=t$ and $t$ is the unique point of coincidence of $f$ and $g$.

Moreover, if $\mathrm{f}$ and $\mathrm{g}$ are weakly compatible, then by Lemma $5, \mathrm{f}$ and $\mathrm{g}$ have a unique common fixed point $t$.

If $\phi(t)=k t, k \in[0,1)$, by Example 8 and Theorem 4 we obtain

Corollary 1 Let $(\mathrm{X}, \mathrm{S})$ be a $\mathrm{S}$ - metric space and $\mathrm{f}, \mathrm{g}: \mathrm{X} \rightarrow \mathrm{X}$ such that

$$
S(f x, f x, f y) \leq k \max \left\{\begin{array}{c}
S(g x, g x, g y), S(g x, g x, f x), S(g y, g y, f y), \\
\frac{S(g y, g y, f x)+S(g x, g x, f y)}{3}
\end{array}\right\}
$$


where $\mathrm{k} \in[0,1)$.

If $\mathrm{f}(\mathrm{X}) \subset \mathrm{g}(\mathrm{X})$ (or $\mathrm{g}(\mathrm{X}) \subset \mathrm{f}(\mathrm{X})$ ) and $\mathrm{g}(\mathrm{X})$ (or $\mathrm{f}(\mathrm{X})$ ) is a complete subspace of $(\mathrm{X}, \mathrm{S})$, then $\mathrm{f}$ and $\mathrm{g}$ have a unique point of coincidence. Moreover, if $\mathrm{f}$ and $\mathrm{g}$ are weakly compatible, then $\mathrm{f}$ and $\mathrm{g}$ have a unique common fixed point.

Example 20 Let $\mathrm{X}=\mathbb{R}$ and $\mathrm{S}(\mathrm{x}, \mathrm{y}, z)=|\mathrm{x}-z|+|\mathrm{y}-z|$. Then $\mathrm{S}(\mathrm{X})$ is a complete $\mathrm{S}$ - metric space. Let $\mathrm{fx}=2 \mathrm{x}-2, \mathrm{gx}=3 \mathrm{x}-4$. Then $\mathrm{f}(\mathrm{X})=\mathbb{R}$, $\mathrm{g}(\mathrm{X})=\mathbb{R}$ and $\mathrm{f}(\mathrm{X}) \subset \mathrm{g}(\mathrm{X})$. If $\mathrm{fx}=\mathrm{gx}$, then $\mathrm{x}=2$ which implies $\mathcal{C}(\mathrm{f}, \mathrm{g})=\{2\}$ and $\mathrm{fg} 2=\mathrm{gf} 2=2$ and $\mathrm{X}=2$ is the unique point of coincidence of $\mathrm{f}$ and $\mathrm{g}$ and $f$ and $g$ are weakly compatible. On the other hand, $S(f x, f x, f y)=4|x-z|$ and $\mathrm{S}(\mathrm{gx}, \mathrm{gx}, \mathrm{gy})=6|x-\mathrm{y}|$. Hence, $\mathrm{S}(\mathrm{fx}, \mathrm{fx}, \mathrm{fy}) \leq \mathrm{kS}(\mathrm{gx}, \mathrm{gx}, \mathrm{gy})$, for $\mathrm{k} \in$ $\left[\frac{2}{3}, 1\right)$. This implies

$$
S(f x, f x, f y) \leq k \max \left\{\begin{array}{c}
S(g x, g x, g y), S(g x, g x, f x), S(g y, g y, f y), \\
\frac{S(g y, g y, f x)+S(g x, g x, f y)}{3}
\end{array}\right\}
$$

for $\mathrm{k} \in\left[\frac{2}{3}, 1\right)$. By Corollary $1, \mathrm{f}$ and $\mathrm{g}$ have a unique common fixed point $x=2$.

If $g(x)=x$, then by Theorem 4 we obtain

Theorem 5 Let $(\mathrm{X}, \mathrm{S})$ be a complete $\mathrm{S}$ - metric space and $\mathrm{f}: \mathrm{X} \rightarrow \mathrm{X}$ such that

$F(S(f x, f x, f y), S(x, x, y), S(x, x, f x), S(y, y, f y), S(y, y, f x), S(x, x, f y)) \leq 0$ for all $\mathrm{x}, \mathrm{y} \in \mathrm{X}$ and some $\mathrm{F} \in \mathcal{F}_{\phi}$.

Then $\mathrm{f}$ has a unique fixed point.

Corollary 2 Let $(\mathrm{X}, \mathrm{S})$ be a complete $\mathrm{S}$ - metric space and $\mathrm{f}: \mathrm{X} \rightarrow \mathrm{X}$ such that

$S(f x, f x, f y) \leq k \max \{S(x, x, y), S(x, x, f x), S(y, y, f y), S(x, x, f y), S(x, x, f y)\}$ for all $\mathrm{x}, \mathrm{y} \in \mathrm{X}$ and $\mathrm{k} \in\left[0, \frac{1}{3}\right]$. Then $\mathrm{f}$ has a unique fixed point.

Proof. The proof follows by Theorem 5 and Example 4 . 
Remark 2 1) By Examples 13 - 19 and Theorem 4 we obtain Theorems 1-7 [13].

2) By Example 4 and Theorem 4 we obtain Corollary 2.19 [13].

3) By Example 5 and Theorem 4 we obtain Theorems 2.2, 2.4 [19] and Theorems 3.2, 3.3, 3.4 [20].

\section{References}

[1] M. Abbas and B. E. Rhoades, Common fixed point results for noncommuting mappings without continuity in generalized metric spaces, Appl. Math. Comput., 215 (5) (2009), 262-269.

[2] J. M. Afra, Fixed point theorems in S - metric spaces, Theory Approx. Appl., 10 (1) (2014), 57-68.

[3] I. Altun and D. Turkoglu, Some fixed point theorems for weakly compatible mappings satisfying an implicit relation, Taiwanesse J. Math., 13 (4) (2009), 1291-1304.

[4] G. V. R. Babu and B. K. Leta, Fixed points of $(\alpha, \psi, \varphi)$ - generalized weakly contractive maps and property $(\mathrm{P})$ in $\mathrm{S}$ - metric spaces, Filomat, 31 (14) (2017), 4469-4481.

[5] B. C. Dhage, Generalized metric space and mappings with fixed point, Bull. Calcutta Math. Soc., 84 (1992), 329-336.

[6] B. C. Dhage, Generalized metric space and topological structures I, An. Ştiinţ. Univ. Al. I. Cuza Iaşi, Mat., 46 (1) (2000), 3-20.

[7] N. V. Dung, N. T. Hieu, S. Radojević, Fixed point theorems for g monotone maps on partially ordered S - metric spaces, Filomat, 28 (9) (2014), 1885-1898.

[8] G. Jungck, Compatible mappings and common fixed points, Int. J. Math. Math. Sci., 9 (1986), 771-779.

[9] J. Matkowski, Fixed point theorems for mappings with a contractive iterate at a point, Proc. Amer. Math. Soc., 62 (2) (1997), 344-348.

[10] Z. Mustafa, Common fixed point of weakly compatible mappings in G metric spaces, Appl. Math. Sci., 92, 6 (2006), 4589-4601. 
[11] Z. Mustafa and B. Sims, Some remarks concerning D - metric spaces, Proc. Conf. Fixed Point Theory Appl., Valencia (Spain), 2013, 189-198.

[12] Z. Mustafa and B. Sims, A new approach to generalized metric spaces, $J$. Nonlinear Convex Anal., 7 (2006), 280-297.

[13] N. Y. Özgür and N. Taş, Some generalizations on fixed point theorems on S - metric spaces, In: Rassias T., Pardalos P. (eds) Essays in Mathematics and its Applications. Springer, Cham, 229-261.

[14] V. Popa, Fixed point theorems for implicit contractive mappings, Stud. Cerc. Stiinţ. Ser. Mat., Univ. Bacău, 7 (1997), 129-133.

[15] V. Popa, Some fixed point theorems for compatible mappings satisfying an implicit relation, Demonstr. Math., 32 (1) (1999), 157-163.

[16] V. Popa, A general fixed point theorem for several mappings in G - metric spaces, Sci. Stud. Res. Ser. Math. Inform., 32 (1) (2011), 205-214.

[17] V. Popa and A.-M. Patriciu, A general fixed point theorem for pairs of weakly compatible mappings in G - metric spaces, J. Nonlinear Sci. Appl., 5 (2) (2012), 151-160.

[18] V. Popa and A.-M. Patriciu, A general fixed point theorem for mappings satisfying an $\phi$ - implicit relation in G - metric spaces, Gazi Univ. J. Sci., 25 (2012), 401-408.

[19] K. Prudhvi, Fixed point results in S - metric spaces, Univer. J. Comput. Math., 3 (2015), 19-21.

[20] K. Prudhvi, Some fixed point results in S - metric spaces, J. Math. Sci. Appl., 4 (1) (2016), 1-3.

[21] S. Sedghi and N. V. Dung, Fixed point theorems on S - metric spaces, Mat. Vesn., 66 (1) (2014), 113-124.

[22] S. Sedghi, N. Shobe and A. Aliouche, A generalization of fixed point theorems in S - metric spaces, Mat. Vesn., 64 (3) (2012), 258-266.

[23] S. Sedghi, M. M. Rezaee, T. Došenović, S. Radenović, Common fixed point theorems for contractive mappings satisfying $\phi$ - maps in $S$ - metric spaces, Acta Univ. Sapientiae, Math., 8 (2) (2016), 298-311. 